\title{
CONTABILIDAD PÚBLICA EN EDUCACIÓN SUPERIOR DEL ECUADOR
}

\section{PUBLIC ACCOUNTING IN ECUADOR'S HIGHER EDUCATION}

\author{
David Richard Pincay-Sancán ${ }^{1}$ \\ Jazmín Elsa Sánchez-Astudillo² \\ José Antonio Flores-Poveda ${ }^{3}$ \\ José Calle-Mejia ${ }^{4}$
}

1. Docente y Director en proyecto de Investigación de la Universidad Estatal de Milagro, Facultad de Ciencias Administrativas y Comerciales. Master en Tributación y Finanzas (Ecuador). E-mail: dav coco@hotmail.com

2. Docente de la Universidad de Guayaquil-Facultad de Ciencias Administrativas y Comerciales, Directora en Proyectos de Investigación UNEMI. Master Administración y Dirección de Empresa (Ecuador). E-mail: Jazmin sanchez4@hotmail.com

3. Profesor Titular de la Universidad de Guayaquil - Facultad de Economía. Master en Tributación y Finanzas (Ecuador). E-mail: florespi@ug.edu.ec

4. Decano de la Facultad de Ciencias Administrativas y Comerciales de la Universidad de Guayaquil. Magister en Docencia Superior (Ecuador). E-mail: Jose.calleme@ug.edu.ec

\section{Citación sugerida:}

Pincay-Sancán, D.R., Sánchez-Astudillo, J.E., Flores-Poveda, J.A. y Calle Mejia, J. (2017). Contabilidad pública en la Educación Superior del Ecuador. 3C Empresa: investigación y pensamiento crítico, 6(4), 29-38. DOI: <http://dx.doi.org/10.17993/3cemp.2017.060432.29-38/>. 


\section{RESUMEN}

Las Normas Internacionales de Contabilidad para el Sector Público NICSP, en Ecuador, tiene la meta de emitir informes financieros al año 2019. Por otro lado las Instituciones de Educación Superior IES, cuentan con 21 facultades públicas que tienen la carrera de CPA o afín. La investigación aborda el uso de las NICSP dentro de las carreras en CPA, metodología aplicada cualitativa-cuantitativa, la herramienta de recolección de datos son la entrevista y encuestas a personal clave dentro de las universidades.

\section{ABSTRACT}

The International Accounting Standards for the Public Sector (IPSAS), in Ecuador, have the goal of issuing financial reports to the year 2019. On the other hand, the Institutions of Higher Education IES, has 21 public schools that have the career of CPA or related. The research addresses the use of IPSAS within careers in CPA, applied qualitative-quantitative methodology, the tool of data collection are the interview and surveys of key personnel within universities.

\section{PALABRAS CLAVE}

Contabilidad Pública, Educación Superior, Informes Financieros, NIC-SP, Universidades.

\section{KEY WORDS}

Public Accounting, Higher Education, Financial Reports, IPSAS, Universities. 


\section{INTRODUCCIÓN}

En América, los países tienen un nuevo marco regulatorio en relación a las normas contables del sector público, se las conoce por sus siglas NIC-SP "Normas Internacionales de Contabilidad para el Sector Publico", emitidas por la Junta de Normas Internacionales de Contabilidad para el Sector Público (IPSASB) (Araya, Caba \& López, 2016).

Marco conceptual basado en el devengo, proyecta al sector público y sus contadores a nivel mundial con visión optimista para la justificación del gasto público-rendición de cuentas (Glöckner, 2016). Este método de registro busca armonizar y unificar la información de los Estados Financieros de las entidades públicas para una rendición de cuentas transparente, en Ecuador el proceso de adopción está fijado en el periodo 2016-2019 (Jorge, Jesus \& Laureano, 2016).

Ecuador tiene como ente regulador de las finanzas públicas, Ministerio de Finanzas, encargado de implementar las fases para adoptar las NIC-SP, y para el Sector Privado la responsabilidad recae en la Superintendencia de Compañías al aplicar NIIF-NIC (SUPERINTENDENCIA, 2009), estos procesos tienen una similitud mediante la transferencia de conocimiento, rol en la que la humanidad interactúa por medio de las Universidades (Echeverría, 2008; Campos, 2007).

El Ministerio de Finanzas para involucrar a las IES en este proceso de aplicación sus primeros pasos ha sido complejo que va desde los altos funcionarios del Ministerio de Finanzas a través de la Subsecretaria de Contabilidad quienes deben entregar el Plan de adaptación a NIC-SP, hasta llegar a los Gobiernos Autónomos Descentralizados y las Universidades, personas relacionadas al Sector Público que deben integrarse a este proceso y seguir puliendo conocimientos y destrezas, buscando siempre el desarrollo y bienestar de los conciudadanos, predispuestos a las capacitaciones y trabajo en equipo hacia este gran reto (Sancán \& Astudillo 2015, July).

En el proceso de implementación, se observa que países como Costa Rica, Chile, Brasil, Colombia, Perú, que están en este proceso avanzan con pasos firmes y concretos, tienen sus normativas, marco conceptual, pero tienen un inconveniente aún les cuesta integrar Información - presupuesto - contabilidad, no así Ecuador trabaja bajo el método de acumulación o devengo desde el año 2000 (Sánchez \& Pincay, 2013), siendo una gran ventaja ya que a través de su herramienta denominada Sistema Integrado de Gestión Financiera (SIGEF) ya integra gran parte de la información (Barahona, 2016).

Las Universidades, "están revisando pensum a fin de incluir las NIC-SP de manera específica como materia de especialización dentro de las carreras de CPA y de manera generalizada en la Carrera de Administración de Empresas, marketing o afines" (Pincay \& Sánchez, 2015).

La investigación se justifica por la necesidad de mejorar en el tratamiento de las Normas Internacionales de Contabilidad para el Sector Públicos que por sus siglas en inglés (IPSAS) dentro de las aulas de clases en las facultades de ciencias administrativas o afines, por un lado la parte administrativa financiera que registra contabilidad gubernamental en sistema Esigef y por otro lado la docencia que transfiere conocimiento por la base del flujo del 
efectivo. A pesar que del modelo NIC-SP está armonizando la información (Roje, Vasicek \& Vasicek, 2010), nuestra aportación proviene del hecho de utilizar transacciones que sean analizados y registradas en los libros diarios como lo exige el marco conceptual publicado el IPSASB en el periodo 2015 (IFAC, 2015; Bisogno \& Tommasetti, 2015). De tal forma que el nuevo profesional CPA, esté en capacidad de registrar, leer y analizar, la información financiera acorde al nuevo método de acumulación, y aplicación en los estados financieros.

Así, el estudio considera como objetivo general analizar el grado de aplicación de las Normas Internacionales de Contabilidad para el Sector Publico NICSP, dentro de las carreras de CPA que se ofertan por parte las universidades públicas del Ecuador.

Adicional complementamos el marco teórico que aborda el nacimiento del devengado, la aplicación de las NICSP y su evolución en el sector administrativo y educativo. Dentro del proyecto de investigación planteado, se han utilizado las Normas Técnicas de Contabilidad Gubernamental emitidas por el Ministerio de Finanzas, las Normas Internacionales de Contabilidad para el Sector Público emitidas por el IPSASB.

\section{METODOLOGÍA}

Una vez revisado el marco teórico, el desarrollo de la investigación empírica realizado en las 30 IES públicas del Ecuador presenta la metodología y resultados para discusión con sus conclusiones limitaciones y propuestas.

Del estudio empírico positivista, aplicamos un enfoque cualitativo, conceptual y revisión de nuevas normas técnicas internacionales aplicadas en la enseñanza del profesional contable. Se revisó la estructura de las mallas curriculares de las facultades que ofertan la carrera de Ingeniería en CPA o afín, además busca profundizar cómo se está preparando el Sector Público para la adaptación de la NIC-SP, ¿Si por medio de adaptación o adopción?, al 2016 existen 21 universidades públicas de 30 que ofertan carreras de Ingeniería en Contaduría Pública, donde aplicamos entrevistas a personeros del Ministerio de Finanzas. Se utilizan normativas legales internacionales y nacionales, herramientas y equipos portátiles, como grabadora, laptops fueron necesarios para el levantamiento de información, que nos conlleva a recabar información necesaria, y analizar la conceptualización para asumir la responsabilidad conforme a estándares internacionales del manejo de la información financiera y su avance dentro de las Universidades.

El acompañamiento de las entrevistas lo realizaron investigadores asociados con más de 20 estudiantes de la carrera de CPA 8vo semestre de la Universidad Estatal de Milagro quien facilito con financiamiento para la movilización. Para entender mejor las preguntas planteadas se utilizaron criterios de 6 expertos académicos en contabilidad, 3 expertos internacionales, entre ellos el Gerente del Banco Mundial para América Latina y el Caribe, a la Subsecretaria de Contabilidad del Ecuador. Los resultados permitieron recoger 11 preguntas que miden el conocimiento referente a las NICSP, la aplicación de las normas internacionales en las aulas de clase, y las disposiciones gubernamentales. 
En la tabla 1, verificamos el trabajo llevado en campo mediante la siguiente ficha técnica.

Tabla 1. Ficha técnica.

\begin{tabular}{|l|l|}
\hline $\begin{array}{l}\text { Ubicación } \\
\text { geográfica }\end{array}$ & Ecuador - Regional \\
\hline Población & 30 universidades públicas en categorías A,B,C, y D \\
\hline $\begin{array}{l}\text { Elemento } \\
\text { muestraL }\end{array}$ & $\begin{array}{l}\text { Informante clave dentro de las universidades, áreas } \\
\text { administrativas y docentes. }\end{array}$ \\
\hline Muestra & $\begin{array}{l}21 \text { universidades, muestreo no probabilístico, por } \\
\text { criterio o juicio }\end{array}$ \\
\hline $\begin{array}{l}\text { Variables } \\
\text { dependiente }\end{array}$ & Facultades de Ciencias Administrativas o comerciales \\
\hline $\begin{array}{l}\text { Levantamiento de } \\
\text { información } \\
\text { apoyo }\end{array}$ & Investigadores asociados y 20 estudiantes de la UNEMI \\
\hline Herramientas & Grabadora, laptops, cámara fotográficas \\
\hline Método utilizado & Encuestas planteadas con 11 preguntas. \\
\hline $\begin{array}{l}\text { Transferencia de } \\
\text { resultados }\end{array}$ & Ponencias nacionales e internacionales, conferencias \\
\hline $\begin{array}{l}\text { Fecha de inicio y } \\
\text { final }\end{array}$ & Julio del 2014 a Septiembre del 2016. \\
\hline
\end{tabular}

Fuente: elaboración propia-2016.

Para la elaboración de las preguntas, fueron considerados criterios de expertos internacionales y docentes en la rama teniendo las siguientes preguntas:

Se pudo denotar que de las 11 preguntas planteadas:

1. ¿Es de vital importancia que las universidades apliquen en su educación las NIC-SP?

2. ¿En la malla curricular de la universidad se ha aplicado la enseñanza de las NIC-SP?

3. ¿La Universidad ha otorgado cursos para capacitar a los estudiantes con respecto a NIC-SP?

4. ¿Cuál es el objetivo de aplicar las NIC-SP?

5. ¿Han realizado conferencias sobre NIC-SP a los estudiantes de la universidad?

6. ¿Han implementado NIC-SP en la elaboración de los Estados Financieros de la Universidad?

7. ¿Cree usted que las NIC-SP son normas que dan calidad a la información contable?

8. ¿Cuál es el criterio que tiene sobre la aplicación de las NIC-SP en las universidades?

9. ¿Cree que Ecuador debe Adoptar o adaptar las NIC-SP?

10. ¿Piensa que la Adopción de las NIC-SP implica mayor eficiencia y efectividad en las auditorías?

11. ¿Con qué fin cree que fueron creadas las NIC-SP? 
Ecuador tiene su proyecto en marcha para la aplicación de las NICSP, la cual se proyecta para el año 2019 en la entrevista realizada a Vicuña (2015) ver anexo 1, nos dice que: "EI objetivo de la aplicación de las NIC-SP es transparentar la información, hacer la comparable con todos los países del mundo, que los inversores vean como están las finanzas públicas del país y hacerla visible a todo el mundo"; palabras tomadas de la entrevista realizada en el año 2015 por nuestros alumnos de la carrera de CPA. El Ministerio de Finanzas a través de su página web da a conocer la información financiera y aspira para el año 2019 presentar sus primeros Estados Financieros con la explicación de normas internacionales (Rodríguez, Navarro \& Alcaraz, 2014).

Todas las universidades a nivel de educación desconocían, sobre la armonización de las NICSP (Benito, Brusca \& Montesinos, 2007), y confundían las NIC del sector público con las del sector privado, esto pone en desventaja al conocimiento de nuestros estudiantes y baja su nivel de aprendizaje, a nivel del personal administrativo solo 5 de ellas afirmaron conocer las NICSP, entre ellas la Universidad Estatal de Milagro, quien ha dictado y participado en este tema de vital importancia.

El Ministerio de Finanzas en Ecuador, aprovecha la ventaja de su experiencia en la integración de la Contabilidad y el Presupuesto con el principio del devengamiento y estima en 5 fases para la aplicación las Normas Internacionales de Contabilidad (Finanzas, 2016), para el Sector Publico (NIC-SP) las cuales se basan en las siguientes:

Tabla 2. Fases de adopción de NICSP en Ecuador.

\begin{tabular}{|l|l|}
\hline Fase 1 & $\begin{array}{l}\text { Generación del Marco Regulatorio, capacitación, creación de grupos de } \\
\text { formadores }\end{array}$ \\
\hline Fase 2 & $\begin{array}{l}\text { Métodos y políticas que a efectuarse en la transición a la nueva normatividad; } \\
\text { creación de grupos institucionales que lideren el proceso. }\end{array}$ \\
\hline Fase 3 & $\begin{array}{l}\text { Proceso de aplicación de la normativa en un año de transición comparable; } \\
\text { evaluando posibles anomalías. }\end{array}$ \\
\hline Fase 4 & $\begin{array}{l}\text { Primer año de convergencia plena, definir indicadores de comparabilidad de } \\
\text { eficiencia, eficacia, economía e iniciar un proceso de gobernanza e } \\
\text { información no financiera. }\end{array}$ \\
\hline Fase 5 & $\begin{array}{l}\text { Evaluación y comparación de los tres años bajo la nueva normatividad. 2019 } \\
\text { primer año con Balances bajo NICSP }\end{array}$ \\
\hline
\end{tabular}

Fuente: Ministerio de Finanzas - 2015.

La adaptación de las NICSP genera un reto al país dentro de las instituciones públicas con particularidad de las empresas públicas ya que ellas son capaces de generar su propio efectivo sin que este dependa de un presupuesto otorgado por el estado, 2019 es la fecha donde las universidades deben estar a la par.

Boyce (2015) nos comenta en la pregunta formulada ¿Han realizado conferencias como Banco Mundial o mediante el IFAC sobre NIC-SP? "Varias conferencias y seminarios. La más recientes, la iniciativa CReCER llevada a cabo en Quito en Mayo, en la segunda Conferencia de la RED de Contadores Públicos de América Latina (FOCAL) en Cartagena de 
Indias en el mes de julio", es evidente que los contadores y docente de esta rama deben mantenerse capacitados y agruparse a este tipo de redes.

También nos comentó a través de esta interrogante planteada durante la investigación ¿EI Banco Mundial de qué manera proyecta la aplicación de las NIC-SP a entidades del Sector Público de manera Internacional? "En el marco de la Estrategia de asistencia técnica a los Gobiernos de los países miembros como parte del fortalecimiento institucional de las instituciones públicas y los sistemas de administración financiera pública. En este contexto, el Banco ofrece servicios de asesoría, entrenamientos e intercambio de conocimientos en la materia" (Boyce, 2015). Analizando esta respuesta por parte del Gerente del Banco Mundial de América Latina, se obtuvo que Ecuador no es un país miembro, sin embargo viene realizando acercamientos de gran importancia, Vicuña (2015), manifestó que se han capacitado los personeros del Ministerio de Finanzas en países como Costa Rica, República Dominicana, Chile y otros que ya están en el proceso de adopción de las NICSP, y para esto Ecuador tiene la ventaja que desde el año 2000 viene aplicando devengado.

En Ecuador, se cuenta con 72 universidades a nivel nacional entre públicas y privadas de las cuales 30 universidades son públicas, es de aquí donde se centró el estudio de la comparación adicional de las mallas curriculares y la propuesta de transformar las IES al complementar las IPSAS (Ramírez, 2014).

Las NIIF en el momento de ser implementadas en el país tuvieron su adecuado proceso al igual que las NIC-SP tendrán hasta su adopción (Benito, Brusca \& Montesinos, 2007); en la actualidad las NIIF se refleja como materia dentro de la carrera de C.P.A., como ejemplo pudimos verificar en una de las instituciones educativas como lo es la Universidad Estatal de Milagro, las NIIF son parte de estudio como materia, que generan créditos alrededor de 3.0 créditos con una totalidad de 6.0 créditos, porque su enseñanza esta aplicada para dos niveles, de la misma manera se espera profundizar las NIC-SP dentro de las mallas curriculares.

\section{CONCLUSIONES}

De las 72 Instituciones de Educación Superior que cuenta Ecuador, 30 de ellas son Universidades y Escuelas Politécnicas Públicas, a estas solo 21 tienen Facultades o Unidades que otorgan el grado de Ingeniería en Contaduría Pública, donde se enfocó el estudio de las NIC-SP y su implementación en la enseñanza contable para armonizar la información entre las leyes, normativas que aplique el Ministerio de Finanzas (Ramírez, 2014).

A través de la entrevista realizada a personero del Ministerio de Finanzas se concluye que el proceso de adaptación de las 37 NIC-SP serán de forma gradual en cinco fases, desde un marco regulatorio hasta la obtención de un primer balance financiero.

La carrera de Contaduría Pública como se lo observa en la tabla 3 esta oferta como licenciatura y como Ingeniería la carrea de CPA, la variación de las estructuras de las mallas es el tiempo de duración de la carrera derivada desde los 8 niveles hasta 10 niveles en distintas universidades, como ejemplo referencial la Universidad Estatal de Milagro cuenta 
con 8 niveles y el título es de Ingeniería en Contaduría Pública Y Auditoria -CPA al igual que la Universidad Nacional De Chimborazo que cuenta con 10 niveles y el título a otorgar es de Ingeniería en Contabilidad Y Auditoria (Espinoza, 2016).

Ubicarnos a través de capacitación en conjunto con el Ministerio de Finanzas, como países Brasil, Chile, Colombia, Perú y Costa Rica en el desarrollo y avances de aplicación de las NICSP y estructurar el plan estratégico definiendo las políticas contables, el marco conceptual, manual de contabilidad integrándose con presupuesto y contabilidad, ventajas que tenemos sobre ellos (Boyce, 2015) .

Cumplir con el objetivo principal de las NIC-SP, una mejor transparencia en la rendición de las cuentas a través de la presentación de los estados financieros (Rodríguez, Navarro \& Alcaraz, 2014), es por este motivo que una vez más se afirma la relevancia que tiene para ser estudiada por los futuros profesionales esta aplicación se espera que se cumpla tanto como en el año de implementación manifestado en el año 2019 o antes de su aplicación ya estén en el pensum académico respectivo (Vicuña, 2015). 


\section{REFERENCIAS BIBLIOGRÁFICAS}

Araya-Leandro, C., Caba-Pérez, M. C., \& López-Hernandez, A. M. (2016). The convergence of the Central American countries to international accounting standards. Revista De Administracao Publica, 50(2), 265-283.

Barahona, Barahona, G. F. (2016). Sistema de contabilidad gubernamental generador de archivos planos para la carga de información financiera AIE-SIGEF del GADP de San Vicente de Pusir, Bolívar Carchi (Bachelor's thesis)

Benito, B., Brusca, I., \& Montesinos, V. (2007). The harmonization of government financial information systems: The role of the IPSASs. International Review of Administrative Sciences, 73(2), 293-317.

Bisogno, M., Santis, S., \& Tommasetti, A. (2015). Public-Sector Consolidated Financial Statements: An Analysis of the Comment Letters on IPSASB's Exposure Draft No. 49. International Journal of Public Administration, 38(4), 311-324.

Boyce, D. (2015) Entrevista sobre las IPSAB al Gerente del Banco Mundial en América Latina. República Dominica-Punta Cana, Proyecto de Investigación 2014-2016 UNEMI (entrevistadora, Sánchez).

Campos, E. B. (2007). La Tercera Misión de la Universidad: El reto de la Transferencia del conocimiento. Revista madri+ d, (41), 2.

Echeverría Ezponda, J. (2008). Transferencia de conocimiento entre comunidades científicas. Arbor, 184(731), 539-548.

Espinoza Cevallos, C. (2016). Calidad de la educación e índices de gestión en relación con el presupuesto de las universidades del Ecuador en el año 2015. Revista Universidad y Sociedad, 8(2), 210-21

Finanzas, De Ministerio. (2016). Normativa de Contabilidad Gubernamental actualizada (A67), recuperado el 30 de septiembre del 2016 a partir de: http://www.finanzas.gob.ec/wpcontent/uploads/downloads/2016/04/Anexo_Acue rdo-Ministerial-067-Normativa-de-Contabilidad-Gubernamental.pdf

García Casella, C. L. (2015). Reemplazo de los conceptos de riqueza e ingreso, no prioritarios en la contabilidad social. Documentos de Trabajo de Contabilidad Social, (1).

Glöckner, A. (2016). New development: The protective role of conservatism in public sector accounting. Public Money and Management, 36(7), 527-530.

Jorge, S. M., Jesus, M. A., \& Laureano, R. M. S. (2016). Governmental accounting maturity toward IPSASs and the approximation to national accounts in the European. 
Ugro, C. W., \& Flores, D. A. (2015). An integrated model for ICT governance and management applied to the council for evaluation, accreditation and quality assurance of higher education institutions in Ecuador (CEAACES). Paper presented at the 2015 International Conference on Computing, Communication and Security, ICCCS 2015.

Nurunnabi, M. (2015). Tensions between politico-institutional factors and accounting regulation in a developing economy: Insights from institutional theory. Business Ethics, 24(4), 398-424.

Pincay, R. D., \& Sanchez, E. J. (2015). Aplicación de las NIIF en las Universidades del Sector Publico. II Congreso Internacional de Contabilidad y Auditoría. UNEMI. Recuperado a partir de: http://media.wix.com/ugd/6202a5 d22a31b3c35e45cfae4ef008ebfd1d68.pdf.ISBN 978-9942-21-382-2 1ra. Edición -Septiembre 2015.

Ramírez, R. (2014). Transformar la universidad para transformar la sociedad. Senescyt.

Rodríguez Bolívar, M. P., Navarro Galera, A., \& Alcaraz Quiles, F. (2014). The improvement of financial accountability for modernization of public sector in emerging countries of Latin america. Reforma y Democracia, 59, 83-118.

Roje, G., Vasicek, D., \& Vasicek, V. (2010). Accounting regulation and IPSAS implementation: Efforts of transition countries toward IPSAS compliance.Journal of Modern Accounting and Auditing, 6(12), 1.

Sancán, D. P., \& Astudillo, J. S. (2015, July). Estudio de las nic-sp y su aplicación en las universidades públicas del ecuador-modelo de convergencia o adaptación (caso universidad estatal de milagro)/study nic-sp and its application in public universities of ecuador-convergence or fitness model (Case Milagro State University). In Global Conference on Business \& Finance Proceedings (Vol. 10, No. 2, p. 1338).

Sánchez, J., \& Pincay, D. (2013). La Contabilidad Pública en América Latina y el Devengo en Ecuador. Analítika: revista de análisis estadístico, (6), 19-32.

Superintendencia, D. C. (2009). Cronograma de Implementación para la Adopción de las NIIF. IFRS para la Elaboración de los Estados Financieros.

Tan, A., Chatterjee, B., Wise, V., \& Hossain, M. (2016). An investigation into the potential adoption of international financial reporting standards in the United States: Implications and implementation. Australian Accounting Review, 26(1), 45-65.

Vicuña, Cevallos, M. (2015). Entrevista aplicación NIC-SP. (Arce, Zambrano, Teran, Cuenca, \& Espinoza, Entrevistadores), 21 de agosto del 2015, Quito - Ecuador. 